\title{
Lighting the cellular fuel gauge
}

A genetically encoded sensor of ATP:ADP ratio reveals the energy status of living mammalian cells.

Balancing high energy demands with production and expenditure of ATP is an art that all mammalian cells have to master, but the details of how they do this are still largely a black box.

So says Gary Yellen, who works in Harvard Medical School's Neurobiology department. He adds that most of what we know about metabolism is encapsulated in textbook chapters and is based on the idea that cells, unless they are in an extreme situation such as starvation, will keep their energy levels relatively high and constant. "That is not something we have data for," he says. "We need to start using reporters that allow monitoring the consumption of ATP in different cells to really nail this down."

Yellen and his team are developing genetically encoded sensors that reveal changes in ATP in living cells. A few years back, the group developed a fluorescent biosensor of ATP:ADP ratio, Perceval, but the sensor would become saturated at the levels of ATP found in mammalian cells.

"We started to optimize the sensor right away after the original version, and when Mathew Tantama, the first author of the paper, came to the lab," Yellen recounts. But the team soon found out that mutations that changed the sensor's sensitivity came with a high price in the amplitude of the signal: as Perceval became less sensitive to ATP, the changes in fluorescence also became smaller. After many rounds of mutagenesis and screening and years of work, they obtained an optimal sensor. PercevalHR (HR for 'high range') senses ATP:ADP ratios expected in mammalian cells yet shows larger signal changes than those of the original sensor. This success was "a tribute to Mathew's perseverance," says Yellen.

Like Perceval, PercevalHR is a ratiometric sensor made of an ATP:ADP ratio-sensing domain from a bacterial protein joined with a circularly permuted fluorescent protein. The ratio of the fluorescence at two different excitation wavelengths is a measure of the occupancy of the sensor by either nucleotide-a signature of the energy status of the cell. The fluorescence signal is independent of the amount of sensor protein or

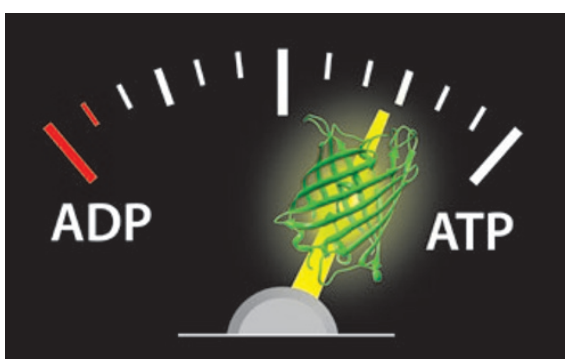

A fluorescence-based sensor of the ATP:ADP ratio is a cellular fuel gauge. Image courtesy of E. Dane.

the absolute cellular concentrations of the nucleotides.

Yellen and his group used the sensor to study changes in ATP:ADP ratio in cultured astrocytes and neurons. They discovered that electrical activity produced changes in the neuron's energetic status. Contrary to the textbook view, Yellen and colleagues were surprised to see that it did not take much to "knock a neuron or astrocyte off its very high ATP:ADP ratio status"; a relatively mild, physiological activation did the trick. "This seems to indicate that cells do allow their energy levels to change, and we think that can play an important role in cellular functions," explains Yellen.

The sensor also responds to two-photon excitation, which means that its use in more physiological preparations, such as brain slices and the in vivo mouse brain, will be relatively straightforward.

One little complication that this sensor has, common to other sensors that rely on fluorescent proteins, is that it is $\mathrm{pH}$-sensitive. Because this can confound the experiments, the group expressed PercevalHR alongside a genetically encoded $\mathrm{pH}$ reporter. In this way, they could correct for the $\mathrm{pH}$-sensitive signal.

PercevalHR allows a wealth of interesting experiments to be performed, such as studying the link between metabolism and neuronal activity. The team is very much focused on neurobiology applications, but researchers in fields such as cancer will also likely find this sensor useful when studying energy levels and their regulation in tumors. One hopes that this sensor will help open the cell's metabolic black box and bring it to light.

\section{Erika Pastrana}

\section{RESEARCH PAPERS}

Tantama, M. et al. Imaging energy status in live cells with a fluorescent biosensor of the intracellular ATPto-ADP ratio. Nat. Commun. 4, 2550 (2013). 